\title{
The Role of Privacy Violations in Privacy Calculus
}

\author{
Maija Poikela \\ Technische Universität Berlin \\ maija.poikela@qu.tu-berlin.de
}

\author{
Sebastian Möller \\ Technische Universität Berlin \\ sebastian.moeller@tu-berlin.de
}

\begin{abstract}
We report a survey study ( $=140)$, assessing various privacy violations that mobile users have experienced, in particular those related with locational privacy. We evaluate the impact of such violations on perceived risks and benefits on usage of location-based applications. Most participants report having experienced some kind of privacy violations; in particular, $\mathbf{7 5 \%}$ report having experienced inappropriate data collection, and $\mathbf{7 4 \%}$ report having received bothersome location-based adverts. Our results suggest that having experienced privacy violations, in particular location-based adverts, might lead to feeling that risks from using location-based applications outweigh the benefits, and have a direct influence on the usage frequency of location-based applications. Our results highlight that locational adverts are not seen as beneficial by the users and may create discomfort, even hindering the adoption of LBS. Nevertheless, the overall benefits of LBS seem to outweigh the risks.
\end{abstract}

\section{INTRODUCTION}

In this age where big data meets machine learning, storing large amounts of personal information from users, as well as processing it, has become affordable and feasible. In practice, this means that privacy violations might have more dire consequences than ever before. An incident involving inappropriate data access today might lead to even more serious consequences later when processed, or even combined with other databases. Data breaches have been found to be costly to the affected companies [1] [2]. In a recent example, a data leak affecting directly 270000 Facebook users resulted in the company's stock market value dropping by nearly $\$ 50$ billion within just two days [3]. Altogether 87 million users had their personal data breached, when the information was passed through those directly affected [4].

Many online and mobile services today are seemingly free for users, relying their income on adverts. The income for the service provider is often based on the "clicks" that the adverts collect. For the service to be beneficial for the service providers, it is important that the advertisement is relevant to the user, which is where behavioral advertising comes to picture. For targeting the adverts meaningfully, they can be based on profiles, which are built based on the users' online behaviors and, in the case of location-based advertising, movement patterns. This creates discomfort in the users, most of whom find targeted advertising invasive [5]. In particular, location-based advertisement creates concern in mobile users [6]. In the context of location-based services (LBS), personal

European Workshop on Usable Security (EuroUSEC) 2018

23 April 2018, London, England

ISBN 1-891562-54-1

https://dx.doi.org/10.14722/eurousec.2018.23015 information in terms of movement patterns can be seen as a commodity that a user exchanges for a personally tailored service. In exchange for these services, including locationbased recommendations, the user may give away information about where they live, work, and spend their freetime.

There is a gap in the literature examining the impact of experienced privacy breaches on users' attitudes with respect to LBS, and the usage of these services. In this work, we aim at filling that gap by presenting results from an online study assessing mobile users' past privacy violations, and their impact on perceived benefits and risks in the usage of locationbased services (LBS), and on their usage frequency.

\section{RELATED WORK}

The effect of privacy violations has been previously studied in an online environment; Smith et al. [7] assessed consumers prior privacy invasions in the online context and found that users who have been exposed to privacy violations have stronger concerns regarding information privacy. Research by Debatin suggest that experienced privacy incidents lead to users changing privacy settings on Facebook, whereas hearing of privacy incidents happening to others does not have similar consequencies [8].

Concerns that users of location-based services have, particularly with respect to disclosure of locational information, were listed in order of importance based on the caused harm by Tsai et al. as: 1. Being stalked, 2. Revealing home location, 3. Being found or intruded by somebody, and 4. Being tracked by the government [9]. Being bothered by locationbased advertisement was mentioned as a scenario that has a high likelihood, but causes little harm. In addition to these, Poikela and Kaiser identified several other risks that users of LBS worry about, including inappropriate secondary use of data, inappropriate data access, theft and identity theft [10]. Perceived benefits in location-based services have been reported by Tsai et al., with the following scenarios rated as the most likely and beneficial: 1. Finding people in an emergency, 2. Keeping track of the location of children and elderly in one's family, 3. Checking people's location to make sure they are ok, and 4. Finding information based on one's location [9]. Lindquist et al. [11]. Sun et al. suggest that perceived benefits on location-based services influence privacy behavior as a second-order construct, including hedonic as well as utilitarian benefits [12].

Through the usage of services and visited locations, very accurate user profiles can be compiled. Based on findings by Culnan et al., users are concerned about user profiling and unsolicited marketing as a consequence of such profiles created of them [13]. Being a subject of behavioral profiling is not 
dissimilar to being under surveillance, and both of these have their consequences on behaviour when a user is aware of the tracking. Sometimes in the privacy literature this is compared with panopticon, a prison construction where the prisoners never knew whether they were being watched or not. The constant possibility of being monitored altered the prisoners' behaviour. Along similar lines, McDonald and Cranor report a chilling effect as a consequence of behavioral advertising, with a significant portion of the users inhibiting their privacy-related behavior when they are aware of data collection by advertisers [5]. Referring to the issue of being under surveillance, Ur et al. report that online behavioral advertising is perceived as "creepy", but at the same time, useful through better matching recommendations [15]. Location-based advertising creates privacy concern in users, inhibiting the acceptance and adoption of the service [14]. Kelley et al. found that context plays a role in how concerned the user is in sharing their location with advertisers [6].

Extant literature suggest that when users engage in privacyrelated behaviors, they first have to assess the risks and the benefits that the behavior in question might entail. This evaluation process is known as privacy calculus [16]. The framework of privacy follows behavioral theories, such as the theory of reasoned action [17], or its later revised version, known as the theory of planned behavior [18], or technology acceptance models [19]. In models for privacy calculus, typically perceived risk is considered a background factor for trust and concern, all of which affect behavior [16], [20].

This paper assesses how typical different privacy violations - in particular with respect to location privacy - are among German mobile users, and the influence that such violations have on privacy calculus, as well as on the usage of locationbased applications. Additionally, we present the various risks and benefits that are perceived in the usage of LBS.

\section{RESEARCH METHOD}

We conducted an online survey with German participants ( $N=141)$ using Limesurvey to study the influence of prior location privacy violations on perceived risks and benefits on location-based applications. Filling out the survey took less than half an hour, for which the participants were given as an incentive an Amazon voucher of $5 €$. The study received an ethical clearance from the Ethics committee of TU Berlin.

\section{A. Demographics}

Altogether 152 participants were recruited through a German online classified advertising service eBay Kleinanzeigen (Kijiji), out of whom 11 did not complete the study. A majority of the respondents were female $(58.5 \%)$, and the mean age was 29.9 years $(S D=9.18)$. In total $90.0 \%$ had completed at least high school level education out of which $42,9 \%$ had also a university level education. More than half of the participants were students or pupils (58.9\%), 30\% were employed, $6.5 \%$ self-employed, and 7.2\% unemployed. More than one in ten $(11.5 \%)$ reported working in an IT-related industry.

\section{B. Measures}

For all measures, we removed the outliers that were more than two standard deviations from the mean.
1) Privacy Violations: We asked the participants if they had experienced privacy violations in the past. Most of these were particular for mobile usage, and additionally, we asked in particular in the context of location information about behavioral advertising, as well as if they knew somebody who has been a victim of a privacy breach. All items measuring privacy violations were measured on a dichotomous scale "yes" (1), or "no" (0). To have an overall measure of having experienced different types of privacy violations, we take a mean value of all the reported privacy violations per participant $(M=.25, S D=.13)$.

(i) Inappropriate Data Collection. First, we asked the participants if they have ever felt discomfort because of data collection that had happened as a consequence of their usage of mobile phone (or online activities). The question was posed this way to identify and record only those incidents of behavioral data collection that have made the participants feel uncomfortable.

(ii) Location-Based Adverts. The category of location-based adverts includes emails, offers, and other adverts based on visited locations. Additionally, we asked participants who expressed having received location-based adverts how they felt about these adverts. This was measured on a fully labelled five-point answer scale as follows: "Very comfortable" (0), "Comfortable" (1), "Neutral" (2), "Uncomfortable" (3), "Very uncomfortable" (4). ( $M=$ 2.92, $S D=.74)$.

(iii) Stalking. We asked whether the participant had ever been a victim of stalking because of their mobile phone use.

(iv) Robbery. We asked whether the participant had ever been a victim of robbery because of their mobile phone use.

(v) Identity Theft. We asked if the participant had ever been a victim of identity theft or impersonation because of their mobile phone use.

(vi) Knowing a Victim of a Location Privacy Violation. Finally, we asked the participants if anyone whom they know have been in a situation where that person?s location privacy has been compromised.

2) Perceived Risks: To measure perceived risks, we used a scale that consists of seven items, asking the users how much they agree with statements regarding various aspects of location-based services posing risks to users. This was measured on a seven-point answer scale, with end labels from "Fully disagree" (0) to "Fully agree" (6). The scale shows good internal consistency (Cronbach's $\alpha=.87$ ). For a total measure of perceived risks, we take a mean value of the seven items $(M=3.71, S D=1.14)$.

3) Perceived Benefits: General benefits that are perceived in using location-based services were measured on a nineitem scale, using a seven-point end-labelled answer scale, anchored with "Fully disagree" (0) and "Fully agree" (6); $M=2.67, S D=1.05$.

4) Privacy Calculus: Finally, we evaluated whether the risks or benefits of using location-based services are considered greater; we measured this using a five-point fully labelled answer scale: "The benefits are much greater than the risks" (0) "The benefits are somewhat greater than the risks" (1), "Benefits and risks are equal" (2), "The risks are somewhat greater than the benefits" (3), "The risks are much greater 
TABLE I. REPORTED PRIVACY VIOLATIONS

\begin{tabular}{|l|c|}
\hline Privacy Violation & Percent of participants \\
\hline Inappropriate data collection & $75.0 \%$ \\
\hline Location-based adverts & $74.3 \%$ \\
\hline Stalking & $4.3 \%$ \\
\hline Robbery & $2.2 \%$ \\
\hline Identity theft & $7.9 \%$ \\
\hline Knowing a victim of a location privavcy violation & $12.2 \%$ \\
\hline Any privacy violation & $92.1 \%$ \\
\hline
\end{tabular}

than the benefits" (4). On average, benefits were considered to slightly outweigh the risks $(M=1.93, S D=1.19)$.

5) LBS Usage Frequency: We measured privacy behavior in this study as self-reported usage frequency of locationbased applications. We asked the participants how often they use various location-based applications. The answers were recorded on a fully labelled four-point scale "Never" (0), "Rarely" (1), "Occasionally" (2), and "Often" (3). A mean value of these were calculated per participant to comprise a measure of LBS usage frequency $(M=.91, S D=.44)$.

\section{RESULTS}

The data that we collected from the survey was predominantly quantitative. We received also some comments regarding the privacy violations, describing incidents of receiving spam, having somebody hacked into their email account, and even having an uncomfortable feeling that their network provider was controlling where they were. In the following, we analyse the quantitative results.

\section{A. Privacy Violations}

In our study, we find that privacy violations are common, $92.1 \%$ of valid responses stated that they have experienced some sort of privacy violations, all of whom stated having experienced them first-hand. Experiencing discomfort because of information that was collected during mobile phone use or from online activities was immensely common; these kind of violations were experienced by a total of $75.0 \%$ of the participants. A similar amount of participants (74.3\%) reported having received adverts based on their location. All other types of privacy violations were reported significantly less (cf. Table I).

The participants who reported having received adverts based on their location mostly reported discomfort; $1.1 \%$ felt "comfortable", 28.3\% "neutral", 47.8\% "uncomfortable", and $22.8 \%$ felt "very uncomfortable". How much discomfort a participant reported having felt correlated with perceived risks in using location-based applications.

Women reported more often uncomfortable information disclosure incidents than men; $t(138)=3.64, p<.001$. Women also felt more discomfort because of location-based adverts; $t(138)=2.14, p=.04$. The results indicated no differences between genders on whether or not location-based adverts were received. Females, and participants with higher education tend to perceive more risks, and feel more discomfort with location-based adverts; however, all these correlations were rather small. These relationships are presented in Table II. No other results were identified based on the demographics.
TABLE II. CORRELATION COEFFICIENTS FOR EDUCATION AND FEELINGS AFTER EXPERIENCED AFTER HAVING EXPERIENCED PRIVACY VIOLATIONS, AND PERCEIVING RISKS AND BENEFITS IN USAGE OF LBS, AS WELL AS THE T-TEST RESULTS FOR THESE CONSTRUCTS BASED ON GENDER. FOR ALL CONSTRUCTS, EXCEPT PERCEIVED BENEFITS, THE MEAN VALUE WAS HIGHER FOR FEMALES.

\begin{tabular}{|l|c|c|}
\hline & Education & Gender \\
\hline $\begin{array}{l}\text { Experienced discomfort with } \\
\text { location-based adverts }\end{array}$ & $r_{s}=.27 *$ & $t(88)=2.36 *$ \\
\hline $\begin{array}{l}\text { Experienced inappropriate data } \\
\text { collection }\end{array}$ & $r_{s}=.22 *$ & $t(135)=3.38 * *$ \\
\hline Perceived risks in LBS & $r_{s}=.28 *$ & $t(135)=2.23 *$ \\
\hline Perceived benefits in LBS & $r_{s}=-.06$ & $t(135)=-2.24 *$ \\
\hline Risks outweigh benefits & $r_{s}=.23 *$ & $t(135)=2.28 *$ \\
\hline
\end{tabular}

We conducted a Spearman's rank-order correlation and found that past privacy violations correlated with an opinion that risks outweigh benefits in the usage of LBS. Additionally, a small positive correlation was found between having experienced inappropriate data collection and perceiving risks in the usage of LBS. Past inappropriate data collection correlated with the feeling that risks in using location-based services exceed the benefits. A statistically significant, albeit very small, negative correlation was found between having experienced inappropriate data collection and perception of benefits in the usage of LBS. For all correlation coefficients, see Table III.

TABLE III. CORRELATION COEFFICIENTS OF EXPERIENCED PRIVACY VIOLATIONS AND PERCEIVED RISKS AS WELL AS BENEFITS. CORRELATIONS SIGNIFICANT AT THE .05 ALPHA LEVEL ARE MARKED WITH ONE ASTERISK $(*)$, AND AT .01 LEVEL WITH TWO ASTERISKS $(* *)$

\begin{tabular}{llll}
\hline & Perceived risks & $\begin{array}{l}\text { Perceived bene- } \\
\text { fits }\end{array}$ & $\begin{array}{l}\text { Risks outweigh } \\
\text { benefits }\end{array}$ \\
\hline $\begin{array}{l}\text { Discomfort with } \\
\text { location-based } \\
\text { adverts }\end{array}$ & $r_{s}=.36 * *$ & $r_{s}=-.22 * *$ & $r_{s}=.17$ \\
\hline $\begin{array}{l}\text { Inappropriate } \\
\text { data collection }\end{array}$ & $r_{s}=.33 * *$ & $r_{s}=-.26 *$ & $r_{s}=.41 * *$ \\
\hline $\begin{array}{l}\text { Past privacy } \\
\text { violations }\end{array}$ & $r_{s}=.32 * *$ & $r_{s}=-.20 *$ & $r_{s}=.34 * *$ \\
\hline
\end{tabular}

\section{B. Perceived Risks}

To understand if some privacy risks are seen as more important than others, we inspect the ratings from each risk item. We find that location-based adverts were seen as the most prevalent risk $(M=4.06, S D=1.64)$, together with inappropriate data access $(M=3.96, S D=1.61)$, and revealing one's home location $(M=3.88, S D=1.80)$. Because a bidirectional answer scale from $0 . .6$ was used, the value three depicts a neutral mean value, for which the participants did not agree nor disagree with the statement that the item in question would be risky. No statistical differences were found between these three items. Because multiple comparisons were conducted, a Bonferroni corrected new alpha level was used; $\alpha=.0024$. The risks are illustrated in the order of importance, measured as the magnitude of perceived risks, in Figure 1. The original item wordings translated into English are reported in the Appendix.

\section{Perceived Benefits}

Next, we evaluated which benefits the users see as the most prevalent. For an illustration, a list of the benefits in the order of importance is depicted in Figure 2. As benefits 


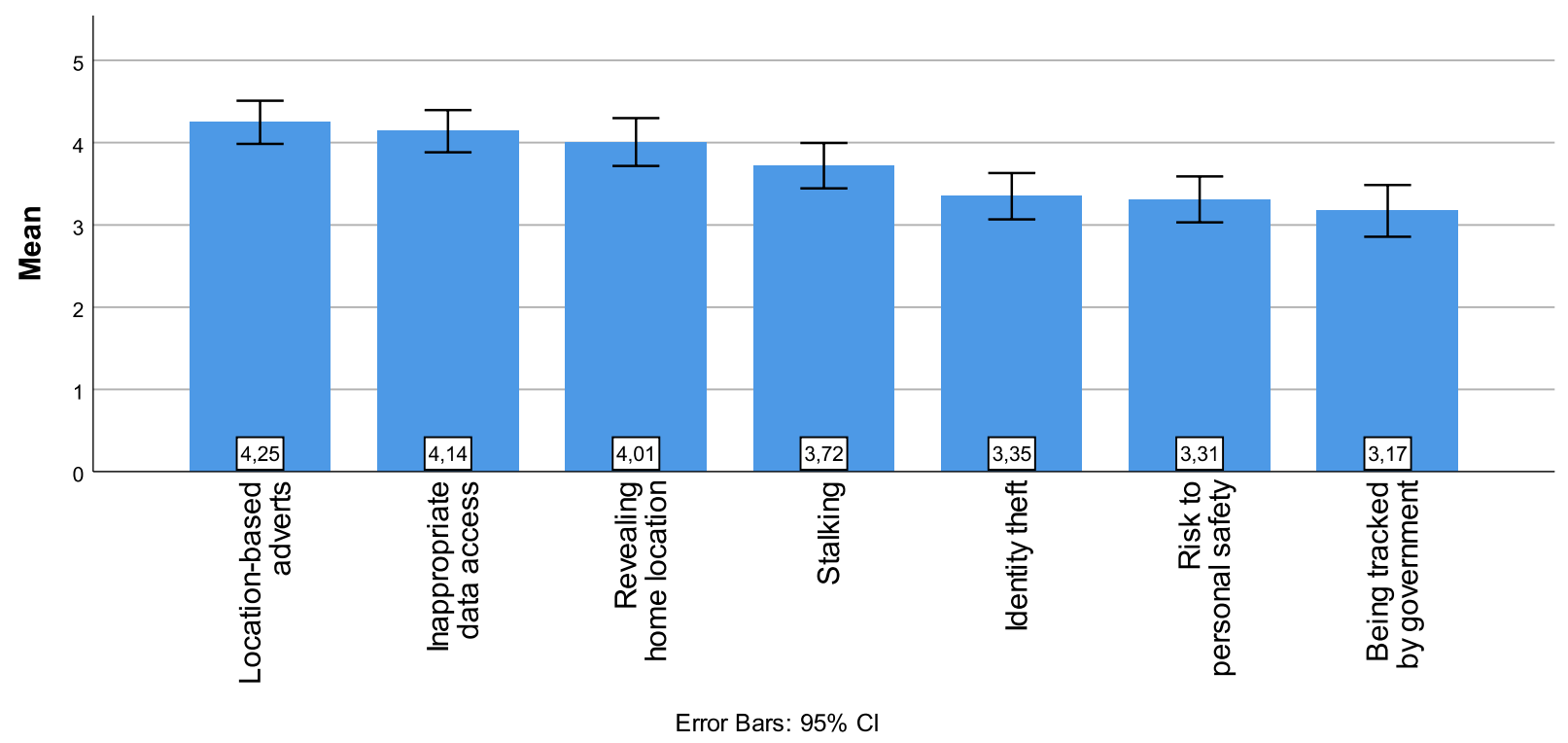

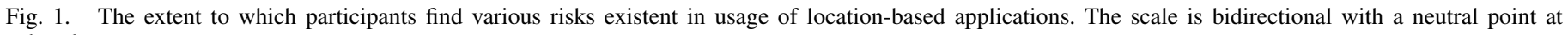
value three.

were reported on a bidirectional seven-point answer scale from zero to six, the value three represents a neutral point, where the statement is not agreed with nor disagreed with. The most important benefit was reported as improved self-representation: using location-based services improve the image that others have of oneself ( $M=3.80, S D=1.83$ ). Furthermore, LBS was stated to make communication easier $(M=3.64, S D=$ 1.60). A statement that LBS would make communication faster was, on average, rather neutral $(M=3.16, S D=1.67)$. There were no statistical differences found between these three most prevalent benefits; the Bonferroni corrected new alpha level considering the multiple paired comparisons was $\alpha=$ .0014. None of the other items were perceived, on average, as beneficial. No statistical differences were found between "facilitated scheduling of meetings" ( $M=2.95, S D=1.80)$, "increased security in society" $(M=2.84, S D=1.73)$, and "improved social life" ( $M=2.76, S D=1.71)$. A list of the benefits in the order of importance is depicted in Figure 2. When participants were asked whether they found the risks or the benefits in the usage of LBS to be greater, the benefits were on average found to outweigh the risks.

\section{Location-Based Advertising}

We analyzed the relationship between the feeling of risks with unsolicited marketing based on locational data, finding benefits in such adverts, and having felt discomfort because of having received them. We found a small correlation between feeling discomfort because of having received location-based adverts, and perception that location-based adverts are risky. Other relationships were statistically significant but very small; all correlation coefficients are listed in Table IV. Finally, we find that there were no differences in risk perception between those who remembered having received them, and those who had not. Similarly, there were no differences found in perceiving benefits in location-based advertising between those who had received them and those who had not.
TABLE IV. CORRELATION COEFFICIENTS OF LOCATION-BASED ADVERTS: DISCOMFORT AFTER HAVING RECEIVED THEM, AND PERCEIVED RISKS AS WELL AS BENEFITS THEREIN. ALL CORRELATIONS ARE SIGNIFICANT AT THE .01 LEVEL.

\begin{tabular}{lll}
\hline & $\begin{array}{l}\text { Perceived risks in } \\
\text { location-based } \\
\text { adverts }\end{array}$ & $\begin{array}{l}\text { Perceived benefits } \\
\text { in location-based } \\
\text { adverts }\end{array}$ \\
\hline $\begin{array}{l}\text { Discomfort with } \\
\text { location-based } \\
\text { adverts }\end{array}$ & $r_{s}=.30 * *$ & $r_{s}=-.27 * *$ \\
\hline $\begin{array}{l}\text { Perceived risks in } \\
\text { location-based } \\
\text { adverts }\end{array}$ & & $r_{s}=-.22 * *$ \\
\hline
\end{tabular}

\section{E. Predicting LBS Usage Frequency}

Next, we ran a multiple linear regression analysis to assess if LBS usage frequency is influenced by the past privacy violations, perceived risks as well as benefits, and by privacy calculus. For the regression analysis, all variables were standardized. We find that the model predicts LBS usage frequency significantly well $(p<.001)$, however, perception of risks does not contribute to the overall model. We ran the analysis again with perceived risks removed. A model with past violations, perceived benefits, and risk-benefit-assessment explains 33\% of the variance in LBS usage frequency $\left(R^{2}=.33, F=20.55\right.$, $p<.01$ ). All of the three independent variables were found to contribute to the LBS usage frequency, with perception of benefits having a stronger influence than past privacy violations, or the feeling that benefits outweigh risks in usage of LBS. The standardized $\beta$-coefficients for all variables are listed in Table V.

\section{DISCUSSION}

We conducted an online study with mobile users in order to assess experienced privacy violations - how typical they are, and what kind of an impact they have on mobile users' attitudes and behavior. 


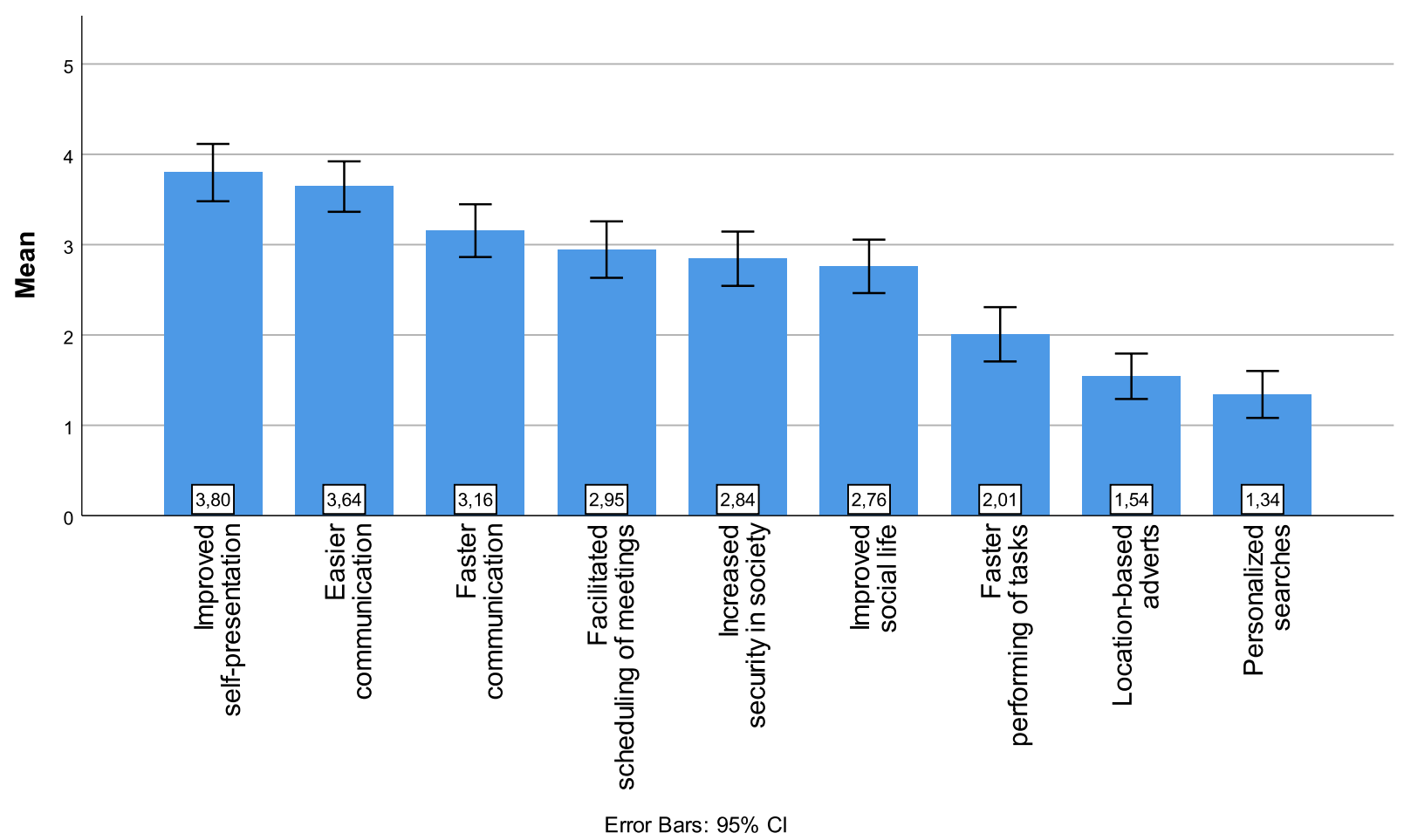

Fig. 2. The perceived benefits of location-based services listed in the order of importance. The items were measured on a bidirectional scale, with value three representing a neutral attitude.

TABLE V. REGRESSION COEFFICIENTS FROM THE MODEL PREDICTING

LBS USAGE FREQUENCY. THE COEFFICIENT SIGNIFICANT AT THE .05 LEVEL IS MARKED WITH AN ASTERISK (*), AND THOSE ON THE .01 LEVEL WITH TWO ASTERISKS $(* *)$.

\begin{tabular}{|c|c|}
\hline & $\beta$ \\
\hline Past privacy violations & $.33^{*}$ \\
\hline Perceived benefits & $.44^{* *}$ \\
\hline Risks outweigh benefits & $-.29 * *$ \\
\hline
\end{tabular}

Nearly all participants in this study reported having experienced some privacy violations $(92.1 \%)$. A majority of the participants (75\%) reported that they had had an incident in the past where they felt discomfort with respect to how their data had been shared. Based on these findings, we can state that privacy violations are extremely commonly experienced among mobile users, including, most importantly, bothersome location-based adverts (experienced by 74\%).

Findings from an earlier interview study [10] identify various risks that users perceive in usage of LBS, reporting the frequency at which different privacy risks are mentioned. We find that the risks that were identified in the interview are also considered problematic by the participants of our online study. However, the order of importance of the privacy risks, as reported in this study, does not seem to match with the order of appearance in the interview study. For example, the most often mentioned risk in the interview was institutional surveillance, referring to the police or the state following one's actions. However, in this study we find that the participants feel almost neutral about the risk of being tracked by the government. This might suggest that the ocurrence of reports of certain risks does not necessarily correspond to the perceived severity of that particular risk. The importance of different perceived risks also does not match with what has been reported earlier by Tsai et al. [9]. This discrepancy could be explained by that the study was conducted when location-based services were not yet highly popular, and their amount has skyrocketed since. Thus, when the study by Tsai et al. was conducted, locationbased services were a rather abstract concept to the users, and the perceived risks would also have been on a hypothetical level. We suggest that our results represent more informed attitudes in that our users are quite likely existing users of these services.

Based on the data from this survey, we identified various different risks that the users of location-based services worry about. At the same time, only two items were rated as beneficial. However, the benefits of using LBS were still rated as greater than the risks. There were several items for which the participants generally did not agree nor disagree that they would be beneficial, including faster communication, facilitated scheduling of meetings, increased security in society, and improved social life. The participants did not consider faster performing of tasks, behavioral adverts, and personalized searches to be benefits that can be achieved through usage of LBS. The benefits that were rated the greatest were improved communication, and improving the image that others have of oneself. This could be achieved for example by posting on social media some selected locations that one visits in order to paint a more desirable picture of oneself. These results could either indicate that these social aspects play a great role - great enough to outweigh the multitude of risks in the usage of LBS - being however inhibited by past privacy violations.

Gender differences were found in feeling discomfort be- 
cause of information disclosure, and feeling discomfort because of location-based adverts: female participants reported more often having experienced discomfort. Women also were found to perceive more risks and less benefits in using locationbased services than men, and reported more often than men that risks of using such services outweigh the benefits. We also found education to correlate with perceiving more risks, and reporting uncomfortable data disclosures. Education had no correlation with perceived benefits in using LBS. No significant relationships were found based on age.

Location based adverts were reportedly received by three quarters of the participants of this study. This number comprises only the participants who have paid attention to the fact that the adverts that they receive are related with their physical location, and could in practice be higher. In earlier works, it has been reported that location-based adverts are sometimes considered useful, in which case they do not cause privacy concern. In this study, the participants who reported having received adverts based on their location felt in the best case neutral about it, but in most cases either uncomfortable or highly uncomfortable. On average, the locationbased adverts were perceived as risky, a result that is in line with previous findings from $\mathrm{Xu}$ et al. [14]. The participants were also particularly asked if they think there are benefits in behavioral advertising; there was a general disagreement with the statement that behavioral adverts based on location would be beneficial, in contrast to the findings of Ur et al., stating that online behavioural advertising is in some cases useful [15]. There were no differences in these attitudes based on whether the participants remembered having received such adverts, suggesting that in general they are perceived as not very useful even if one receives them. Also, receiving such adverts does not seem to affect the risks perceived therein in either direction. These findings question the usefulness of location-based advertising - if mobile users do not find them useful irrespective of whether they receive them or not, and feel rather anxious about them because of the possible risks, it is questionable whether they are useful to either party - mobile users at the receiving end, or the advertisers.

The users who had privacy violations perceived less benefits in using location-based applications, however, this correlation was nearly negligible. Having experienced inappropriate disclosure of information correlated with perception of risks in using location-based systems, as well as with an opinion that risks outweigh the benefits in using LBS. Based on these results, we postulate that past privacy violations have an influence on privacy calculus - evaluation of risks as well as benefits on location-based services. This evaluation in turn has an influence on reported usage of location-based applications. Therefore, it is not only in the best interest of the mobile users to have the incidents that can be considered as privacy violations minimized, but also the application manufacturers should be interested in the topic, as the usage of their products is directly influenced by it.

\section{A. Limitations}

The generalizability of our results is somewhat limited because, even though this was an online study, the participants were recruited from a limited geographical area. In the context of a privacy study, the self-selection bias also causes issues, as the most privacy concerned are likely not to take part in such surveys, or might drop out if the questions feel too intrusive.

\section{CONCLUSION}

In this paper we report a study assessing prior privacy violations experienced by individuals. We find that incidents causing discomfort, such as inappropriate data disclosure, or receiving bothersome location-based adverts, are common. We suggest that these violations have an impact on how useful, and how risky the usage of location-based applications is perceived. Experienced privacy violations leave the individuals with feelings of discomfort, and together with perceived benefits, influence the usage of location-based applications. Our results highlight that location-based adverts are not perceived as beneficial, but rather as a risk to one's privacy. Past privacy violations, with location-based adverts being the most commonly reported privacy incident, seem to also affect adoption of location-based services. This raises a question whether behavioral adverts are particularly useful for the users, or even for the service providers.

\section{REFERENCES}

[1] C. T. Pierson, "Data Breaches Highlight the Importance of Privacy.," Financial Executive, 2009.

[2] A. Acquisti, A. Friedman, and R. Telang, "Is There a Cost to Privacy Breaches? An Event Study - viewcontent.cgi," International Conference on Information Systems (ICIS), 2006.

[3] A. Eric and D. Ingram, "Cambridge Analytica CEO claims influence on U.S. election, Facebook questioned," 2018.

[4] S. Frier, "Facebook Just Doubled the Number of People Exposed in Data Breach," apr 2018.

[5] A. M. Mcdonald and L. F. Cranor, "Beliefs and Behaviors : Internet Users ' Understanding of Behavioral Advertising," 38th Research Conference on Communication Information and Internet Policy Telecommunications Policy Research ConferenceOctober 2 2010, pp. 1-31, 2010.

[6] P. G. Kelley, M. Benisch, L. F. Cranor, and N. Sadeh, "When Are Users Comfortable Sharing Locations with Advertisers ?," Human Factors, 2011.

[7] H. J. Smith, S. J. Milberg, and S. J. Burke, "Information Privacy: Measuring Individuals' Concerns about Organizational Practices," MIS Quarterly, vol. 20, no. 2, pp. 167-196, 1996.

[8] B. Debatin, J. P. Lovejoy, A. K. Horn, and B. N. Hughes, "Facebook and online privacy: Attitudes, behaviors, and unintended consequences," Journal of Computer-Mediated Communication, 2009.

[9] J. Y. Tsai, P. Kelley, P. Drielsma, L. F. Cranor, J. Hong, and N. Sadeh, "Who's viewed you?: The impact of feedback in a mobile locationsharing application," Proceedings of the 27th international conference on Human factors in computing systems - CHI 09, p. 2003, 2009.

[10] M. Poikela and F. Kaiser, "“It Is a Topic That Confuses Me" - Privacy Perceptions in Usage of Location-Based Applications," in European Workshop on Usable Security, (Geneva), Internet Society, 2016.

[11] J. Lindqvist, J. Cranshaw, J. Wiese, J. Hong, and J. Zimmerman, "I'm the mayor of my house," in Proceedings of the 2011 annual conference on Human factors in computing systems CHI 11, p. 2409, 2011.

[12] Y. Sun, N. Wang, X. L. Shen, and J. X. Zhang, "Location information disclosure in location-based social network services: Privacy calculus, benefit structure, and gender differences," Computers in Human Behavior, 2015.

[13] M. J. Culnan and P. K. Armstrong, "Information Privacy Concerns, Procedural Fairness, and Impersonal Trust: An Empirical Investigation,” 1999.

[14] H. Xu, X. Luo, J. M. Carroll, and M. B. Rosson, "The personalization privacy paradox: An exploratory study of decision making process for location-aware marketing," Decision Support Systems, vol. 51, no. 1, pp. 42-52, 2011. 
[15] B. Ur, P. G. Leon, L. F. Cranor, R. Shay, and Y. Wang, "Smart, useful, scary, creepy," in Proceedings of the Eighth Symposium on Usable Privacy and Security - SOUPS '12, 2012.

[16] T. Dinev and P. Hart, "An extended privacy calculus model for ecommerce transactions," Information Systems Research, vol. 17, no. 1, pp. 61-80, 2006.

[17] I. Ajzen and M. Fishbein, "Theory of Reasoned Action," 1980.

[18] I. Ajzen, "The theory of planned behavior," 1991.

[19] V. Venkatesh, M. G. Morris, G. B. Davis, and F. D. Davis, "USER ACCEPTANCE OF INFORMATION TECHNOLOGY: TOWARD A UNIFIED VIEW.," MIS Quarterly, vol. 27, no. 3, pp. 425-478, 2003.

[20] T. Dinev, M. Bellotto, P. Hart, V. Russo, I. Serra, and C. Colautti, "Privacy calculus model in e-commerce - a study of Italy and the United States," 2006.

\section{APPENDIX}

The following scales have been translated into English for reporting. For each scale, the question order was randomized for each participant. The items that were reverse-coded prior to analysis in order to match with the scale direction are marked with an asterisk (*).

\section{A. Risk Perception Scale}

Q. Please take a moment to answer the following questions concerning you and your stance to the risks of using locationbased services. Please use the full scale to indicate your preference.

1) Using location-based applications involves the risk of getting stalked.

2) I am worried that using location-based applications would lead to my home location being revealed.

3) I am worried that using location-based applications involves the risk of becoming a victim of identity theft.

4) I am worried that if I use location-based applications, strangers might know too much about my activities.

5) Using location-based applications poses a threat to my personal safety.

\section{B. Benefit Perception}

Q. Please answer the following questions using the full scale.

1) The use of location-based services makes communication faster.

2) Location-based services create a secure society.

3) Using location-based services makes it easy to schedule meetings.

4) Location-based services improve my social life.

5) The use of location-based services allows me to perform tasks faster.

6) Location based services are beneficial for personalized searches.

7) Location-based services are beneficial for behavior-based advertising.

8) The use of location-based services makes communication easier.

9) Using location-based services improves the impression others have of me.

\section{Past Privacy Violations}

1) Have you ever felt discomfort because of collected data that you shared using a mobile device or doing online activities?

2) Have you ever received e-mails, offers or advertising based on a location you visited?

3) How did you feel as a consequence of the e-mails, offers, or adverts that you received based on a location you visited?

- Very comfortable

- Comfortable

- Neither comfortable nor uncomfortable

- Uncomfortable

- Very uncomfortable

4) Have you ever been a victim of the following because of your mobile phone usage? Please select all that apply.
a Stalking
b Robbery
c Identity theft or impersonation

5) Has anyone you know been in a situation where this person's locational privacy was compromised?

\section{Privacy Calculus}

Q. How do you assess the benefits and risks of using location-based services?

- The benefits are much greater than the risks.

- The benefits are somewhat greater than the risks

- The benefits and risks are equal.

- The risks are somewhat greater than the benefits.

- The risks are much greater than the benefits. 